\title{
6-Demethoxynobiletin, a Nobiletin-Analog Citrus Flavonoid, Enhances Extracellular Signal-Regulated Kinase Phosphorylation in PC12D Cells
}

\author{
Junko Kimura, ${ }^{a}$ Kiyomitsu Nemoto, ${ }^{a}$ Akihito Yokosuka, ${ }^{b}$ Yoshihiro Mimaki, ${ }^{b}$ \\ Masakuni Degawa, ${ }^{a}$ and Yasushi Ohizumi ${ }^{*, a, c, d}$ \\ ${ }^{a}$ Department of Molecular Toxicology, School of Pharmaceutical Sciences, University of Shizuoka; 52-1 Yada, \\ Suruga-ku, Shizuoka 422-8526, Japan: ${ }^{b}$ Laboratory of Medicinal Plant Science, School of Pharmacy, Tokyo \\ University of Pharmacy and Life Sciences; 1432-1 Horinouchi, Hachioji, Tokyo 192-0392, Japan: ${ }^{c}$ Department of \\ Anti-dementia Functional Food Development, Research Center of Supercritical Fluid Technology, Graduate School \\ of Engineering, Tohoku University; 6-6-07 Aoba, Aramaki, Aoba-ku, Sendai 980-8579, Japan: and ${ }^{d}$ Laboratory of \\ Kampo Medicines, Yokohama College of Pharmacy; 601 Matano-cho, Totsuka-ku, Yokohama 245-0066, Japan. \\ Received March 19, 2013; accepted August 1, 2013; advance publication released online August 9, 2013
}

We previously demonstrated that nobiletin, a polymethoxylated flavone isolated from citrus peels, has the potential to improve cognitive dysfunction in patients with Alzheimer's disease (AD). Recent studies suggest that the generation of intraneuronal amyloid-beta $(\mathrm{A} \beta)$ oligomers is an early event in the pathogenesis of AD. A $\beta$ oligomers cause deficits in the regulation of the extracellular signal-regulated kinase (ERK) signaling which is critical for consolidation of the memory. Our previous studies revealed that nobiletin activated ERK signaling and subsequent cyclic AMP response element-dependent transcription. In this study, the effects of five nobiletin analogs, 6-demethoxynobiletin, tangeretin, 5-demethylnobiletin, sinensetin, and 6-demethoxytangeretin, isolated from citrus peels were assessed on ERK phosphorylation in PC12D cells, and the structure-activity relationships were examined. PC12D cells were treated with nobiletin or its analogs, and the cell extracts were analyzed by Western blotting using an antibody specific to phosphorylated ERK. 6-Demethoxynobiletin markedly enhanced ERK phosphorylation in a concentration-dependent manner. These results may be useful in developing drugs and functional foods using citrus peels for the treatment of dementia including AD. disease

Key words flavonoid; 6-demethoxynobiletin; nobiletin; extracellular signal-regulated kinase; Alzheimer's

Alzheimer's disease (AD) is an age-related neurodegenerative disorder characterized by progressive memory impairment and cognitive decline. Furthermore, intraneuronal soluble amyloid-beta $(\mathrm{A} \beta)$ oligomers have been shown to cause cognitive deficits in AD model animals. ${ }^{1,2)}$ Moreover, it is suggested that the accumulation of $\mathrm{A} \beta$ oligomers is an early event in the pathogenesis of $\mathrm{AD}$ and it triggers neuronal cell dysfunction. ${ }^{3)}$ Long-term potentiation (LTP), a form of synaptic plasticity, is believed to underlie learning and memory in the hippocampus. ${ }^{4)}$ The cAMP-dependent signaling pathway plays a crucial role in hippocampal LTP. ${ }^{5,6)}$ The extracellularsignal regulated kinase (ERK) activates cAMP response element (CRE)-binding protein (CREB), a multifunctional transcription factor, which is known as the ERK/CREB cascade. Activation of ERK in mammalian hippocampus is important in consolidation of the memory. ${ }^{7,8)}$ On the other hand, $\mathrm{A} \beta$ oligomers inhibit ERK activation and subsequently CREB in human neuroblastoma cells and primary neurons, which is prevented by an $\mathrm{A} \beta$ oligomer-specific antibody. ${ }^{9)}$

Previously, we successfully identified nobiletin, a polymethoxylated flavone found in citrus peels, as a natural compound with a unique mechanism of neurotrophic action, markedly activating protein kinase A/mitogen-activated protein kinase (MAPK)/ERK/CREB signaling and CREdependent transcription in cell culture systems including cultured rat hippocampal neurons. ${ }^{10,11)}$ Our previous studies on the nobiletin analogs 6-demethoxynobiletin, tangeretin, 5-demethylnobiletin, sinensetin, and 6-demethoxytangeretin

The authors declare no conflict of interest. revealed that the compounds activate CRE-dependent transcription in the following order: nobiletin $>>6$-demethoxynobiletin>tangeretin. ${ }^{11)}$ In addition, it has been reported that 6-demethoxynobiletin as well as nobiletin suppress the production of matrix metalloprotease-9/gelatinase $B$, which is involved in cartilage destruction and rheumatoid arthritis. ${ }^{12)}$ However, the nobiletin analogs abilities to activate intracellular signaling in PC12D cells (a subclone of PC12 cells) are still unknown.

In this study, we examined whether nobiletin analogs enhance ERK phosphorylation and clarified the structure-activity relationships of these analogs using nobiletin as a reference compound. To the best of our knowledge, this is the first study to report that 6-demethoxynobiletin enhances ERK phosphorylation in PC12D cells.

\section{MATERIALS AND METHODS}

Preparation of Nobiletin and Its Analogs Nobiletin and its analogs 6-demethoxynobiletin, tangeretin, 5-demethylnobiletin, sinensetin, and 6-demethoxytangeretin (Fig. 1) were isolated from the peels of Citrus depressa HAYATA as previously described previously. ${ }^{11,13)}$ These compounds were dissolved in dimethyl sulfoxide (DMSO, Sigma-Aldrich Co., LLC, U.S.A.) and stored at $-20^{\circ} \mathrm{C}$.

Cell Culture PC12D cells were cultured in high-glucose Dulbecco's modified Eagle's medium supplemented with $10 \%$ heat-inactivated horse serum (HS; Life Technologies Corp.), $5 \%$ heat-inactivated fetal bovine serum (FBS; MP Biomedicals LLC), 4 mm L-glutamine (Sigma-Aldrich Co., LLC), peni- 
<smiles>[R2]c1cc(-c2cc(=O)c3c([R2])c([R2])c(OC)c([R3])c3o2)ccc1OC</smiles>

1, Nobiletin

2, 5-Demethylnobiletin

3, Tangeretin

4, Sinensetin

5, 6-Demethoxytangeretin

6, 6-Demethoxynobiletin

$\begin{array}{cccc}\mathbf{R}_{\mathbf{1}} & \mathbf{R}_{\mathbf{2}} & \mathbf{R}_{\mathbf{3}} & \mathbf{R}_{\mathbf{4}} \\ \mathrm{Me} & \text { OMe } & \text { OMe } & \text { OMe } \\ \mathrm{H} & \text { OMe } & \text { OMe } & \text { OMe } \\ \mathrm{Me} & \text { OMe } & \text { OMe } & \mathrm{H} \\ \mathrm{Me} & \text { OMe } & \mathrm{H} & \text { OMe } \\ \mathrm{Me} & \mathrm{H} & \text { OMe } & \mathrm{H} \\ \mathrm{Me} & \mathrm{H} & \text { OMe } & \text { OMe }\end{array}$

Fig. 1. Chemical Structure of Nobiletin and Its Analogs Isolated from C. depressa PeELS

cillin, and streptomycin (Meiji Seika Pharma Co., Ltd.). Cells were maintained in a humidified atmosphere containing $5 \%$ $\mathrm{CO}_{2}$ at $37^{\circ} \mathrm{C}$ as described previously. ${ }^{14)}$

Western Blotting PC12D cells were plated at a density of $5 \times 10^{5}$ cells $/ 35-\mathrm{mm}$ dish and incubated in the culture medium for $48 \mathrm{~h}$. The culture medium was replaced with fresh low-serum medium (1\% heat-inactivated FBS and 2\% heat-inactivated HS) containing 30, 50, or $100 \mu \mathrm{M} 6$-demethoxynobiletin, tangeretin, 5-demethylnobiletin, sinensetin, and 6-demethoxytangeretin, or nobiletin (reference compound) or $<0.3 \%$ DMSO (vehicle control). After stimulation, cells were lysed with lysis buffer as described previously. ${ }^{15,16)}$ Protein assay was performed using the $\mathrm{BCA}^{\mathrm{TM}}$ Protein Assay Reagent (Thermo Fisher Scientific, Inc.). Western blotting was performed as described previously. ${ }^{11,15)}$ The blotted membrane was blocked with TBST buffer $(150 \mathrm{~mm} \mathrm{NaCl}, 0.1 \%$ Tween 20 , $10 \mathrm{~mm}$ Tris- $\mathrm{HCl}, \mathrm{pH} 7.6)$ containing 5\% skim milk for $1 \mathrm{~h}$ at room temperature. Thereafter, the membrane was incubated with phospho-ERK1/2 (Thr202/Tyr204) antibody in 5\% BSA/ TBST buffer overnight at $4{ }^{\circ} \mathrm{C}$ and subsequently incubated with horseradish peroxidase (HRP)-conjugated anti-rabbit immunoglobulin $\mathrm{G}(\mathrm{IgG})$ for $1 \mathrm{~h}$ at room temperature. Following antibody stripping, the membrane was reprobed with p44/42 MAPK (Erk1/2) antibody in 5\% BSA/TBST buffer overnight at $4^{\circ} \mathrm{C}$ and subsequently incubated with HRP-conjugated antirabbit $\mathrm{IgG}$ for $1 \mathrm{~h}$ at room temperature. All antibodies were obtained from Cell Signaling Technology, Inc., U.S.A. Immunoreactivity was detected using the SuperSignal ${ }^{\circledR}$ West Pico Chemiluminescent Substrate (Thermo Fisher Scientific, Inc.).

Statistical Analysis Statistical analysis was performed using GraphPad Prism (GraphPad Software). Statistically significant differences were evaluated by one-way analysis of variance followed by Tukey's multiple comparison test. (a)

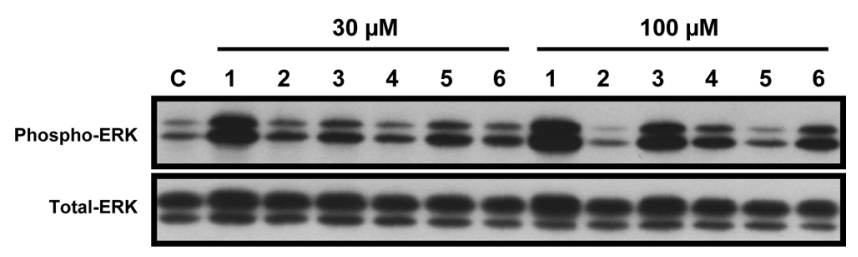

(b)

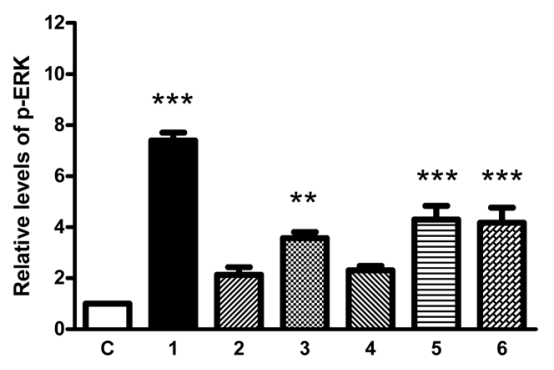

(c)

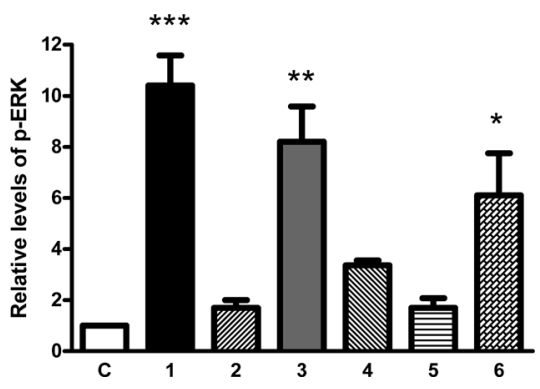

Fig. 2. Effects of Nobiletin and Its Analogs on ERK Phosphorylation in PC12D Cells (a)

Cells were stimulated with nobiletin or its analogs $(30$ or $100 \mu \mathrm{m})$ for $5 \mathrm{~min}$. Western blotting was performed using anti-phospho-ERK1/2 antibody. The membranes were then stripped and reprobed with anti-ERK1/2 antibody. The relative levels of phosphorylation evoked by the compounds at 30 and $100 \mu \mathrm{m}$ are presented in (b) and (c), respectively. Similar results were obtained from at least three independent experiments. Lanes: C, vehicle control; 1, nobiletin; 2, 5-demethylnobiletin; 3, tangeretin; 4, sinensetin; 5, 6-demethoxytangeretin; and 6, 6-demethoxynobiletin. The values represent the mean \pm S.E.M. $(n=3) . *_{p}<0.05, * * p<$ $0.01, * * * p<0.001$ versus vehicle control.

\section{RESULTS}

Nobiletin analogs were evaluated for their ability to stimulate ERK phosphorylation in PC12D cells (Fig. 2). 6-Demethoxynobiletin, 6-demethoxytangeretin, and tangeretin promoted ERK phosphorylation at $30 \mu \mathrm{M}$ (Figs. 2a, b), whereas at $100 \mu \mathrm{M}, 6$-demethoxynobiletin and tangeretin markedly evoked ERK phosphorylation in the following order: nobiletin $>$ tangeretin $>6$-demethoxynobiletin (Figs. 2a, c).

The peak of the effect of nobiletin on ERK phosphorylation has been reported to occur at approximately $5 \mathrm{~min}{ }^{10)}$ In this experiment, we examined the time-dependent effects of nobiletin analogs over a 15-min time course. 6-Demethoxynobiletin and tangeretin enhanced ERK phosphorylation in a concentration-dependent manner (Fig. 3a). Tangeretin induced ERK phosphorylation in a time-dependent manner, and this effect peaked at $5 \mathrm{~min}$. On the other hand, the effect induced 
(a)
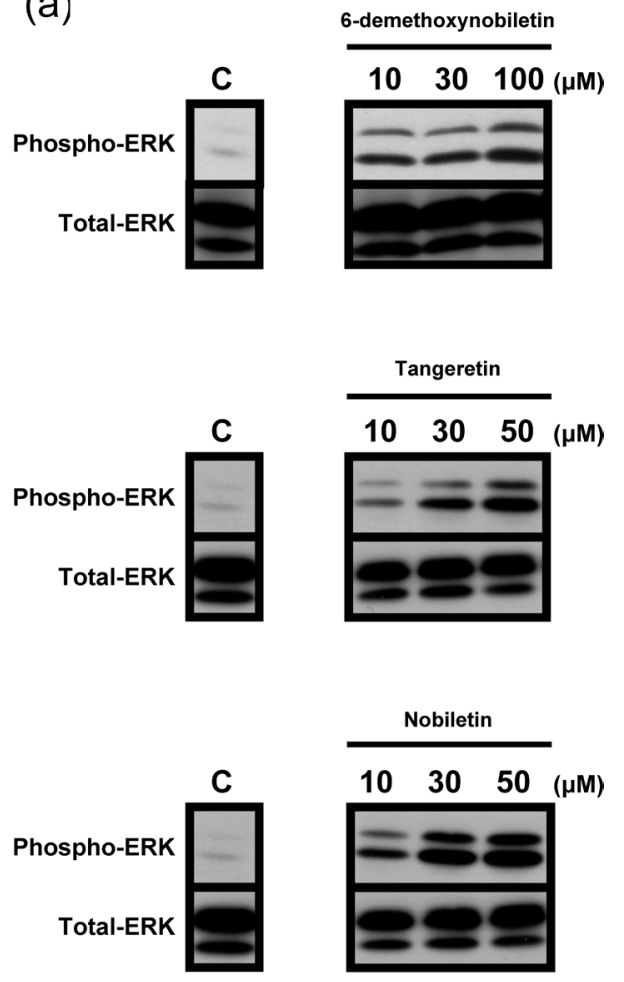

(b)
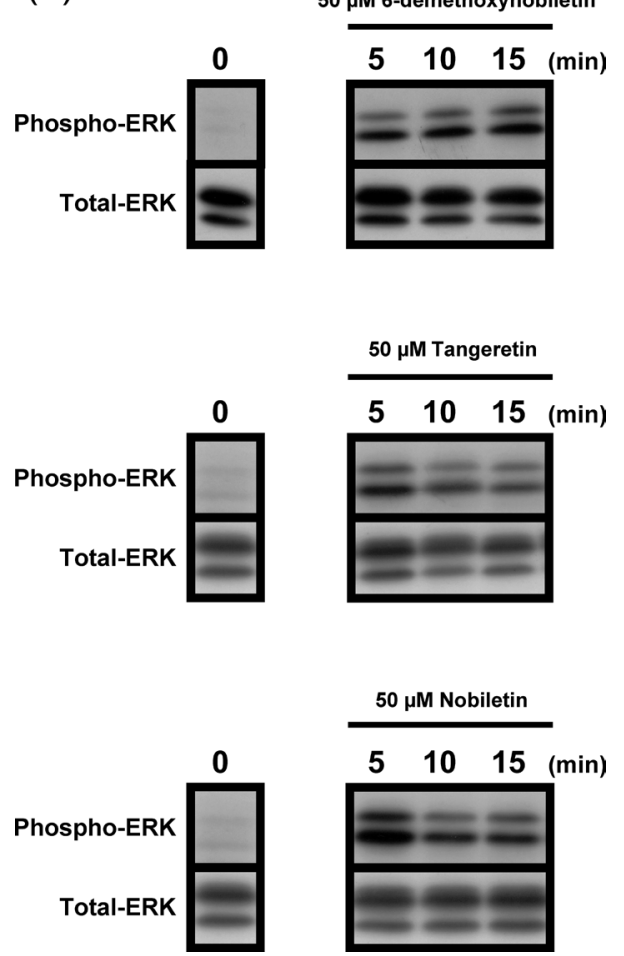

Fig. 3. Nobiletin, Tangeretin, and 6-Demethoxynobiletin Enhanced ERK Phosphorylation in a Concentration-Dependent Manner (a)

PC12D cells were stimulated with nobiletin $(10,30$, and $50 \mu \mathrm{M})$, tangeretin $(10$, 30 , and $50 \mu \mathrm{M})$, 6-demethoxynobiletin $(10,30$, and $100 \mu \mathrm{m})$, or vehicle control $(0.3 \%$ DMSO; lane C) for $5 \mathrm{~min}$. (b) Time-dependent effects of nobiletin, tangeretin, and 6-demethoxynobiletin on ERK phosphorylation. The cells were stimulated with $50 \mu \mathrm{M}$ nobiletin, tangeretin, or 6-demethoxynobiletin for the indicated times. ERK phosphorylation was detected by Western blotting using anti-phospho-ERK1/2 and anti-ERK $1 / 2$ antibodies. by 6-demethoxynobiletin was sustained until 15 min (Fig. 3b).

\section{DISCUSSION}

ERK, a type of MAPK, has been implicated as an important component in a large number of signaling systems involved in hippocampal synaptic plasticity and hippocampaldependent memory formation, ${ }^{7)}$ whereas $\mathrm{A} \beta$ oligomers are known to downregulate ERK and CREB phosphorylation in human neuroblastoma cells and primary neurons. ${ }^{9)}$

Nobiletin stimulates ERK phosphorylation, resulting in the stimulation of CREB phosphorylation and CRE-mediated transcription in PC12D cells. ${ }^{10)}$ Moreover, we previously demonstrated that nobiletin improves learning and memory impairment in transgenic $\mathrm{AD}$ model mice, ${ }^{17)} \mathrm{AD}$ model rats, $\left.{ }^{15}\right)$ olfactory bulbectomized mice, ${ }^{18)}$ and cerebral ischemia mice. ${ }^{19)}$ Taken together, these findings suggest that nobiletin is a promising candidate for the treatment of AD.

Our previous experiments demonstrated that nobiletin induced ERK phosphorylation in a concentration- and timedependent manner in PC12D cells. ${ }^{10)}$ Furthermore, Furukawa et al. demonstrated the ability of tangeretin to activate ERK in rat neuron cultures. ${ }^{20)}$ To the best of our knowledge, this is the first study to demonstrate that 6-demethoxynobiletin evokes ERK phosphorylation in a concentration-dependent manner in PC12D cells (Figs. 2, 3). It is possible that 6-demethoxynobiletin acts on the same pathway as that of nobiletin.

6-Demethoxynobiletin and its analogs evoked phosphorylate ERK at $100 \mu \mathrm{M}$ in the following order: nobiletin $>$ tangeretin $>6$-demethoxynobiletin (Fig. 2c). Considering these results, methoxyl substituents at the 5,6,8, and $3^{\prime}$ positions were proposed to be important for their ability to evoke ERK phosphorylation.

In addition, our previous studies demonstrated that PC12D cells treated with nobiletin or its analogs displayed the characteristic morphology of neurite outgrowth at $48 \mathrm{~h}$ after treatment in the following order: nobiletin $>>6$-demethoxynobiletin. ${ }^{11)}$ In this study, PC12D cells treated with nobiletin and 6-demethoxynobiletin for $24 \mathrm{~h}$ exhibited clear morphological changes (data not shown). These compounds probably exert a neurotrophic factor-like effect on PC12D cells within $24 \mathrm{~h}$ after treatment.

It is noteworthy that three compounds activated ERK phosphorylation and CRE-dependent transcription and that there was a correlation between them, suggesting that they may additively and/or synergistically activate CREB-mediated transcription. Although sinensetin activated CRE-dependent transcription, ${ }^{11)}$ its ability to stimulate ERK phosphorylation was weak in PC12D cells (Fig. 2). This finding raises the possibility of an alternative pathway for CRE-dependent transcription in the action of sinensetin.

It has been demonstrated that the contents of nobiletin were 22 and $3.6 \mathrm{mg} / \mathrm{kg}$, corresponding to approximately 55 and $8.9 \mu \mathrm{m}$, respectively, in the brains of mice that were intraperitoneally and orally administered $50 \mathrm{mg} / \mathrm{kg}$ nobiletin, respectively. ${ }^{21)}$ These results suggest that nobiletin may quickly penetrate the blood-brain barrier of mice, and the in vivo concentrations of nobiletin in the brain are sufficient to activate ERK signaling. There is a possibility that the behavior of 6-demethoxynobiletin and other analogs is similar to that of nobiletin. 
Biological evaluation of 6-demethoxynobiletin activities such as its effects on the CREB signaling pathway is ongoing. Furthermore, it is necessary to demonstrate the effect of 6-demethoxynobiletin in animal models with memory impairment.

In conclusion, 6-demethoxynobiletin, similar to nobiletin, is a potentially useful compound in the development of functional foods for treating various forms of dementia including AD.

Acknowledgments This work was supported by Grants for the research projects "Application in Promoting New Policy of Agriculture, Forestry, and Fisheries" and "Development of fundamental technology for analysis and evaluation of functional agricultural products and functional foods" from the Ministry of Agriculture, Forestry, and Fisheries, Japan.

\section{REFERENCES}

1) Lesné S, Koh MT, Kotilinek L, Kayed R, Glabe CG, Yang A, Gallagher M, Ashe KH. A specific amyloid-beta protein assembly in the brain impairs memory. Nature, 440, 352-357 (2006).

2) Oddo S, Caccamo A, Shepherd JD, Murphy MP, Golde TE, Kayed R, Metherate R, Mattson MP, Akbari Y, LaFerla FM. Triple-transgenic model of Alzheimer's disease with plaques and tangles: intracellular A $\beta$ and synaptic dysfunction. Neuron, 39, 409-421 (2003).

3) LaFerla FM, Green KN, Oddo S. Intracellular amyloid-beta in Alzheimer's disease. Nat. Rev. Neurosci., 8, 499-509 (2007).

4) Bliss TV, Collingridge GL. A synaptic model of memory: long-term potentiation in the hippocampus. Nature, 361, 31-39 (1993).

5) Impey S, Mark M, Villacres EC, Poser S, Chavkin C, Storm DR. Induction of CRE-mediated gene expression by stimuli that generate long-lasting LTP in area CA1 of the hippocampus. Neuron, 16, 973-982 (1996).

6) Abel T, Nguyen PV, Barad M, Deuel TA, Kandel ER, Bourtchouladze R. Genetic demonstration of a role for PKA in the late phase of LTP and in hippocampus-based long-term memory. Cell, 88, 615-626 (1997).

7) Sweatt JD. Mitogen-activated protein kinases in synaptic plasticity and memory. Curr. Opin. Neurobiol., 14, 311-317 (2004).

8) Chwang WB, Arthur JS, Schumacher A, Sweatt JD. The nuclear kinase mitogen- and stress-activated protein kinase 1 regulates hippocampal chromatin remodeling in memory formation. $J$. Neurosci., 27, 12732-12742 (2007).

9) Ma Q-L, Harris-White ME, Ubeda OJ, Simmons M, Beech W, Lim GP, Teter B, Frautschy SA, Cole GM. Evidence of A $\beta$ - and transgene-dependent defects in ERK-CREB signaling in Alzheimer's models. J. Neurochem., 103, 1594-1607 (2007).

10) Nagase H, Yamakuni T, Matsuzaki K, Maruyama Y, Kasahara J, Hinohara Y, Kondo S, Mimaki Y, Sashida Y, Tank AW, Fukunaga K, Ohizumi Y. Mechanism of neurotrophic action of nobiletin in PC12D cells. Biochemistry, 44, 13683-13691 (2005).
11) Nagase H, Omae N, Omori A, Nakagawasai O, Tadano T, Yokosuka A, Sashida Y, Mimaki Y, Yamakuni T, Ohizumi Y. Nobiletin and its related flavonoids with CRE-dependent transcription-stimulating and neuritegenic activities. Biochem. Biophys. Res. Commun., 337, 1330-1336 (2005).

12) Ishiwa J, Sato T, Mimaki $Y$, Sashida $Y$, Yano M, Ito A. A citrus flavonoid, nobiletin, suppresses production and gene expression of matrix metalloproteinase 9/gelatinase B in rabbit synovial fibroblasts. J. Rheumatol., 27, 20-25 (2000).

13) Mimaki Y, Sashida Y, Furuya S, Sakagami H. Polymethoxylated flavonoids from the peels of Citrus depressa. Natural. Med., 54, 351 (2000).

14) Li P, Matsunaga K, Yamakuni T, Ohizumi Y. Nardosinone, the first enhancer of neurite outgrowth-promoting activity of staurosporine and dibutyryl cyclic AMP in PC12D cells. Brain Res. Dev. Brain Res., 145, 177-183 (2003).

15) Matsuzaki K, Yamakuni T, Hashimoto M, Haque AM, Shido O, Mimaki Y, Sashida Y, Ohizumi Y. Nobiletin restoring beta-amyloid-impaired CREB phosphorylation rescues memory deterioration in Alzheimer's disease model rats. Neurosci. Lett., 400, 230-234 (2006).

16) Al Rahim M, Nakajima A, Misawa N, Shindo K, Adachi K, Shizuri Y, Ohizumi Y, Yamakuni T. A novel diol-derivative of chalcone produced by bioconversion, 3-(2,3-dihydroxyphenyl)-1-phenylpropan-1-one, activates PKA/MEK/ERK signaling and antagonizes A $\beta$-inhibition of the cascade in cultured rat CNS neurons. Eur. $J$. Pharmacol., 600, 10-17 (2008).

17) Onozuka H, Nakajima A, Matsuzaki K, Shin R-W, Ogino K, Saigusa D, Tetsu N, Yokosuka A, Sashida Y, Mimaki Y, Yamakuni T, Ohizumi Y. Nobiletin, a citrus flavonoid, improves memory impairment and $\mathrm{A} \beta$ pathology in a transgenic mouse model of Alzheimer's disease. J. Pharmacol. Exp. Ther., 326, 739-744 (2008).

18) Nakajima A, Yamakuni T, Haraguchi M, Omae N, Song S-Y, Kato C, Nakagawasai O, Tadano T, Yokosuka A, Mimaki Y, Sashida Y, Ohizumi Y. Nobiletin, a citrus flavonoid that improves memory impairment, rescues bulbectomy-induced cholinergic neurodegeneration in mice. J. Pharmacol. Sci., 105, 122-126 (2007).

19) Yamamoto Y, Shioda N, Han F, Moriguchi S, Nakajima A, Yokosuka A, Mimaki Y, Sashida Y, Yamakuni T, Ohizumi Y, Fukunaga $\mathrm{K}$. Nobiletin improves brain ischemia-induced learning and memory deficits through stimulation of CaMKII and CREB phosphorylation. Brain Res., 1295, 218-229 (2009).

20) Furukawa $Y$, Okuyama S, Amakura $Y$, Watanabe S, Fukata T, Nakajima M, Yoshimura M, Yoshida T. Isolation and characterization of activators of ERK/MAPK from Citrus plants. Int. J. Mol. Sci., 13, 1832-1845 (2012)

21) Saigusa D, Shibuya M, Jinno D, Yamakoshi H, Iwabuchi Y, Yokosuka A, Mimaki Y, Naganuma A, Ohizumi Y, Tomioka Y, Yamakuni T. High-performance liquid chromatography with photodiode array detection for determination of nobiletin content in the brain and serum of mice administrated the natural compound. Anal. Bioanal. Chem., 400, 3635-3641 (2011). 